九州大学学術情報リポジトリ

Kyushu University Institutional Repository

\title{
On the invariant multiple decision procedures
}

Kudo, Akio

Indian Statistical Institute | Kyushu University

https://doi.org/10.5109/12968

出版情報 : 統計数理研究. 6 (3/4)，pp. 57-68，1956-03. Research Association of Statistical Sciences

バージョン：

権利関係 : 


\title{
ON THE INVARIANT MULTIPLE DECISION PROCEDURES
}

\author{
By \\ Akio Kuvô \\ (Received, March 15, 1956)
}

\$1. Introduction. The notion of invariance seems to have some importance in the theory of multiple decision problems. The invariance seems to be a sort of substitute to the similarity, which plays an important role in the classical theory of test of significance according to the NEYMANPEARSON set up.

The purpose of this paper is to present a theorem which shows that the condition of invariance is a stronger one than it usually appears to be, and to present a theorem which may be considered to be a sort of analogue to the NEYMAN-PEARSON's fundamental lemma. We shall also show that a unified treatment of the slippage tests so far obtained is possible by making use of this theorem, and we shall give some new results.

The section 2 is devoted to the statement and the proof of the result concerning the nature of the invariant tests. In the section 3 we shall give a theorem which is a generalization of a theorem previously obtained by the author (KuDô [3]) concerning the PEARSON. CHANDRASEKAR's statistics. In the section 4 , we shall discuss the optimum slippage tests for gamma distribution, which is believed to be new and may be useful in practical situations.

The author would like to express his hearty thanks to Professor T. KitAGawa for the suggestions and criticisms given by him.

$\S 2$. On the invariant decision procedure. There seems to be three types of invariance so far defined in the current literatures on decision function theory (for instance [4], [5]). Let $R$ be a sample space with sample point $x$, and let $\varphi(x)$ be a decision function defined on the sample space. Further let $G=\{g\}$ be a group of transformations $g$ defined on $R$. We further assume that there is defined a complete measure $\mu$ on the sample space $R$.

The three types of invariance are as follows.

Definition 2.1. $\varphi(x)$ is said to be invariant under the transformation group

$\left(1^{\circ}\right)$ in the strict sense if $\varphi(g x)=\varphi(x)$ for all $x$ and $g$.

$\left(2^{\circ}\right)$ in the usual sense if $\varphi(g x)=\varphi(x)$ for all $x$ and $g$ except possibly for a set $N$ of $x$ with measure zero. 
$\left(3^{\circ}\right)$ in the generalised sense if $\varphi(g x)=\varphi(x)$ for all $x$ and $g$ except possibly for sets $N_{g}$ of $x$ with measure zero, where $N_{g}$ may vary from $g$ to $g$ and $\bigcup_{g \in G^{*}} N_{g}$ may not be of measure zero.

The first definition was adopted in the papers by PAulson [5], Traux [7] and KUDÔ [3], which can be, as we shall see later, generalised to the case of $\left(2^{\circ}\right)$.

Here let us confine ourselves to the case where $R$ is an $N$-dimensional euclidean space $R^{N}=\left\{\underset{\sim}{x} ; \underset{\sim}{x}=\left(x_{1}, x_{2}, \ldots, x_{N}\right)\right\}$.

We can now consider various types of transformation groups on this $N$-dimensional euclidean space. However, we shall here restrict ourselves to the class of transformations by which $\underset{\sim}{x}=\left(x_{1}, x_{2}, \ldots, x_{N}\right)$ is transformed to $\left(a x_{1}+b, a x_{2}+b, \ldots, a x_{N}+b\right)$

Definition 2.2. The transformation group (A), (B), (C), (D) and (E) on the space $R$ are defined to be the totality of such transformations as $\left(x_{1}, x_{2}, \ldots, x_{N}\right)$ to $\left(a x_{1}+b, a x_{2}+b, \ldots, a x_{N}+b\right)$, where $a$ and $b$ are all possible constants satisfying the conditions
(a)
$a \neq 0$
(b)
$\infty>a>0$
(c)
$a \neq 0, \quad b=0$
(d)
$\infty>a>0, \quad b=0$
(e)
$a=1, \quad \infty>b>-\infty$

respectively.

It is quite clear that we are justified to call these (A), (B), (C), (D) and $(E)$ as groups, for each of them forms a group in the usual sense. It may be noticed here that we need not require $a$ and $b$ to be constants, and we have

Definition 2.3. The transformation groups $\left(\mathrm{A}^{\prime}\right),\left(\mathrm{B}^{\prime}\right),\left(\mathrm{C}^{\prime}\right),\left(\mathrm{D}^{\prime}\right)$ and $\left(\mathrm{E}^{\prime}\right)$ on the space $R$ are defined to be totality of such transformations as $\left(x_{1}\right.$, $\left.x_{2}, \ldots, x_{N}\right)$ to $\left(a x_{1}+b, a x_{2}+b, \ldots, a x_{N}+b\right)$, where a and $b$ are all possible functions of $\underset{\sim}{x}=\left(x_{1}, x_{2}, \ldots, x_{N}\right)$ satisfying the conditions (a), (b), (c), (d) and (e) respectively for all $x$, and the transformation groups $\left(\mathrm{A}^{\prime \prime}\right),\left(\mathrm{B}^{\prime \prime}\right)$, $\left(\mathrm{C}^{\prime \prime}\right),\left(\mathrm{D}^{\prime \prime}\right)$ and $\left(\mathrm{E}^{\prime \prime}\right)$ are the same satisfying these conditions except possibly for a set of measure zero which is independent of the individual functions, and finally the transformation groups $\left(\mathrm{A}^{\prime \prime \prime}\right),\left(\mathrm{B}^{\prime \prime \prime}\right),\left(\mathrm{C}^{\prime \prime \prime}\right),\left(\mathrm{D}^{\prime \prime \prime}\right)$ and $\left(\mathrm{E}^{\prime \prime \prime}\right)$ are the same satisf ying these conditions except possibly for a set of measure zero which may vary from an individual function to another.

Concerning the relation of these four types of transformation groups, we observe that, for instance, $\left(\mathrm{A}^{\prime \prime \prime}\right)$ contains $\left(\mathrm{A}^{\prime \prime}\right),\left(\mathrm{A}^{\prime}\right)$ and $(\mathrm{A})$ as their subgroups, and it is obvious that if $\varphi(\underset{\sim}{\boldsymbol{x}})=\varphi\left(\boldsymbol{x}_{1}, \ldots \boldsymbol{x}_{N}\right)$ is invariant under transformation group, say $\left(\mathrm{A}^{\prime}\right)$ or $\left(\mathrm{A}^{\prime \prime}\right)$ or $\left(\mathrm{A}^{\prime \prime \prime}\right)$, it is also invariant under 
the transformation group $(\mathrm{A})$. Concerning the invariance under these transformation groups, we have the following relation, which is believed to be new and has not been found in any of the papers for far as the author is aware of, and is enunciated in the following

Theorem 2.1. The necessary and sufficient conditions for $\varphi(\underset{\sim}{x})$ to be invariant under each one of the transformation groups $(A),(B),(C),(D)$ and $(E)$ in the sense of $\left(1^{\circ}\right)$ or $\left(2^{\circ}\right)$ is that it is invariant under the transformation groups $\left(A^{\prime \prime \prime}\right),\left(B^{\prime \prime \prime}\right),\left(C^{\prime \prime \prime}\right),\left(D^{\prime \prime \prime}\right)$ and $\left(E^{\prime \prime \prime}\right)$ in the same sense respectively.

Proof. We shall prove the equivalence of invariance in the sense of $\left(2^{\circ}\right)$ under each one of the transformation groups $(\mathrm{A})$ and $\left(\mathrm{A}^{\prime \prime \prime}\right)$ only, as we can prove the same for other cases in exactly the same way. For this

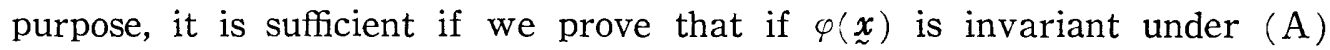
it is also invariant under $\left(\mathrm{A}^{\prime \prime \prime}\right)$.

If $\varphi(\underset{\sim}{x})$ be invariant under (A) but not under $\left(\mathrm{A}^{\prime \prime \prime}\right)$, then there exist

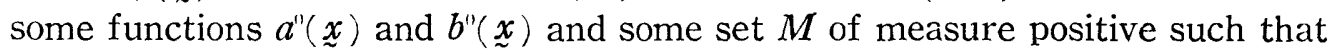

$$
\begin{aligned}
& \varphi\left(x_{1}, x_{2}, \ldots, x_{N}\right)
\end{aligned}
$$

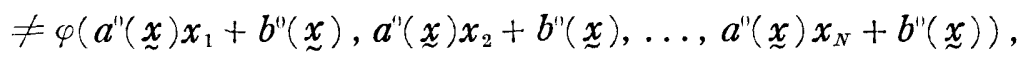

for all $x$ not belonging to $M$. Let $N$ be a set of measure zero defined by (2), in Definition 2.1, namely the set of measure zero, except for whose points the condition of invariance under $(\mathrm{A})$ in the sense of $\left(2^{\circ}\right)$ should hold true. Then there must be at least one point $\stackrel{\sim}{0}^{0}=\left(x_{1}^{0}, x_{:}^{0}, \ldots, x_{N}^{n}\right)$ which belongs to $M$ but not $N$, for which we should have

$$
\begin{aligned}
& \varphi\left(x_{1}^{\prime \prime}, x_{2}^{\prime \prime}, \ldots, x_{N}^{\prime \prime}\right) \\
& \quad \neq \varphi\left(a^{\prime \prime}(\underbrace{}_{x_{1}}) x_{1}^{\prime \prime}+b^{\prime \prime}\left(\underline{x}^{\prime \prime}\right), a^{\prime \prime}\left(\underline{\sim}^{\prime \prime}\right) x_{2}^{\prime \prime}+b^{\prime \prime}(\underbrace{\prime \prime}_{\sim}), \ldots, a^{\prime \prime}\left(\underline{x}^{\prime \prime}\right) x_{N}^{\prime \prime}+b^{\prime \prime}\left(\underline{x}^{\prime \prime}\right)\right) .
\end{aligned}
$$

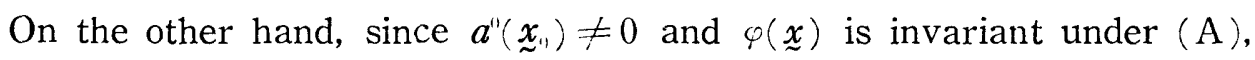

$$
\begin{aligned}
& \varphi\left(x_{1}, x_{2}, \ldots, x_{N}\right) \\
& =\varphi\left(a^{\prime \prime}\left(\underline{x}^{\prime \prime}\right) x_{1}+b^{\prime \prime}\left(\underline{x}^{\prime \prime}\right), a^{\prime \prime}(\underset{x}{x}) x_{2}+b^{\prime \prime}(\underset{\sim}{x}), \ldots, a^{\prime \prime}\left(\underline{x}^{\prime \prime}\right) x_{N}+b^{\prime \prime}\left(\underline{x}^{\prime \prime}\right)\right)
\end{aligned}
$$

should hold true for all $\underset{x}{x}$ not belonging to $N$ and also in the case when $\underline{x}=\underline{x}^{0}$, which leads us to a contradiction. Therefore the measure of set $M$ must be zero.

This theorem reveals us that the condition of invariance under a transformation group like (A), (B) etc. is a stronger condition than it usually appears to be.

There do exist, however, practical situations where we are intuitively sure that according to whatever the scale and the original point, namely the location, the observation may be made, we should be lead to the same conclusion, and in this case we are justified to restrict curselves to the class 
of decision functions invariant under the transformation group (B). This would be only the justification for confining ourselves to a class of invariant decision procedures such as defined above.

The multiple decision procedure may be formulated as follows. Suppose we are interested in making either of $M$ decisions, say, $D_{i}(i=1,2, \ldots, M)$ on the basis of observations, say $x$. Let $d_{i}(x)(i=1,2, \ldots, M)$ denote the probabilities of making decisions $D_{i}(i=1,2, \ldots, M)$ respectively after observing $\underset{x}{x}$. Naturally these $M$ functions must satisfy the conditions

$$
0 \leqq d_{i}(\underline{x}) \leqq 1,(i=1,2, \ldots M), \sum_{i=1}^{M} d_{i}(x)=1
$$

and to meet our mathematical necessity we assume $d_{i}(\underset{\sim}{\boldsymbol{x}})$ are all measurable functions.

According to the formulation of the statistical decision function due to $H$. WALD [8], we shall call the vector valued function $\underset{d}{d}(x)=\left(d_{1}(x)\right.$, $\left.\boldsymbol{d}_{2}(\underset{\sim}{\boldsymbol{x}}), \ldots, \boldsymbol{d}_{N}(\underset{\boldsymbol{x}}{\boldsymbol{x}})\right)$ the decision function which expresses multiple decision procedure formulated as above. If all the $d_{i}(x)$ 's are invariant under the same transformation group in a sense, this decision function is said to be invariant in the same way.

In this formulation, we are sometimes interested in the following problem. Let $D_{i}$ be the decision that the observation $\underset{x}{x}$ has come from a population with a frequency function $f_{i}(\tilde{x})$, and we are interested in finding out the invariant decision function which maximizes

$$
\sum_{i=1}^{M} a_{i} \int_{R^{N}} d_{i}(x) f_{i}(x) d x,
$$

where $a_{i}(i=1,2, \ldots, M)$ are some positive constants.

In the case when we do not require the condition of invariance, the solution would be the decision functions which satisfy the following condition except for a set of measure zero.

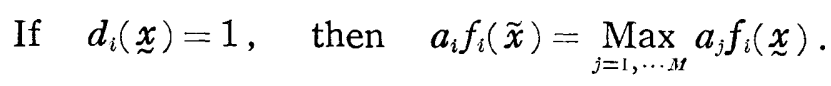

However the solution would be quite defferent if we assume the condition of invariance. The answer for certain types of invariance are enunciated in the following.

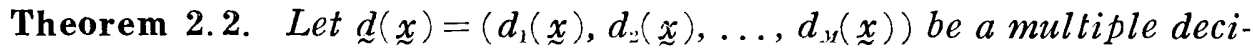
sion proceoure, $f_{i}(\underline{x})(i=1,2, \ldots, M)$ be frequency functions, and $a_{i}(i=$ $1,2, \ldots, M)$ be positive constants. Then the decision function which maximizes the integral

$$
\sum_{i=1}^{M} a_{i} \int_{R^{N}} d_{i}(\underline{x}) f_{i}(\underline{x}) d \underline{x}
$$

among all the decision functions invariant in the sense of $\left(1^{\circ}\right)$ or $\left(2^{\circ}\right)$ 
under the transformation group $(A),(B),(C),(D)$ and $(E)$ must satisfy the following conditions except for a set of measure zero,

$$
\text { If } d_{i}(\underset{\sim}{x})=1 \quad \text { then } a_{i} g_{i}(y)=\operatorname{Max}_{j=1, \ldots, y} a_{j} g_{j}(y),
$$

where $g_{j}(y)$ and $y$ are defined by

$$
\begin{aligned}
& \left\{\begin{aligned}
\underset{\sim}{y}= & \left(y_{1}, y_{2}, \ldots, y_{N-2}\right), \\
& y_{i}=\left(x_{i}-x_{N}\right) /\left(x_{N-1}-x_{N}\right) \quad(i=1,2, \ldots, N-2) \\
g_{j}(\underset{\sim}{y})= & \int_{x=-\infty}^{\infty} \int_{z=-\infty}^{\infty}|x|^{N-2} f_{j}\left(y_{1} x+z, \ldots, y_{N-2} x+z, x+z, z\right) d x d z
\end{aligned}\right. \\
& \left\{\begin{aligned}
\underset{\sim}{y}= & \left(y_{1}, y_{2}, \ldots, y_{N-2}\right), \\
& y_{i}=\left(x_{i}-x_{N}\right) /\left|x_{N-1}-x_{N}\right| \quad(i=1,2, \ldots, N-2) \\
g_{j}(\underset{\sim}{y})= & \int_{x=1}^{\infty} \int_{z=-\infty}^{\infty} x^{N-2} f_{j}\left(y_{1} x+z, \ldots, y_{N-2} x+z, x+z, z\right) d x d z
\end{aligned}\right.
\end{aligned}
$$

$$
\left\{\begin{aligned}
\underset{\sim}{y} & =\left(y_{1}, y_{2}, \ldots, y_{N-1}\right), \quad y_{i}=x_{i} / x_{N} \quad(i==1,2, \ldots, N-1) \\
g_{j}(\underset{\sim}{\boldsymbol{y}}) & =\int_{x=-\infty}^{\infty} x^{N-1} f_{j}\left(y_{1} x, \ldots, y_{N-1} x, x\right) d x
\end{aligned}\right.
$$

$$
\left\{\begin{aligned}
\underset{\sim}{y} & =\left(y_{1}, \ldots, y_{N-1}\right), \quad y_{i}=x_{i} /\left|x_{N}\right| \quad(i=1,2, \ldots, N-1) \\
g_{j}(\underset{\sim}{y}) & =\int_{x=0}^{\infty} x^{N-1} f_{j}\left(y_{1} x, \ldots, y_{N-1} x, x\right) d x
\end{aligned}\right.
$$

$$
\left\{\begin{aligned}
\underset{\sim}{y} & =\left(y_{1}, y_{2}, \ldots, y_{N-1}\right), y_{i}=x_{i}-x_{N} \quad(i=1,2, \ldots, N-1) \\
g_{j}(\underset{\sim}{y}) & =\int_{x=-\infty}^{\infty} f_{j}\left(y_{1}+x, y_{2}+x, \ldots, y_{N-1}+x, x\right) d x
\end{aligned}\right.
$$

respectively, provided the functions $g_{j}(y)$ exist in each case.

Proof. We shall give the proof for the case of transformation group (B) only. Other cases can be proved in exactly the same way.

At first let us devide $R^{N}$ into three disjoint part $R_{N}(+), R_{N}(0)$ and $R_{N}(-)$ according to $x_{N-1}>x_{N}, x_{N-1}=x_{N}$ and $x_{N-1}<x_{N}$.

It is clear that these three subsets are invariant under the transformation group (B) in the sense that any point in either of these three subsets is transformed into the same subset.

On the other hand $R_{N}(0)$ is of measure zero, and hence the integral can be split into two parts, one is the integral on $R^{N}(+)$, the other on $R^{N}(-)$. Here let us observe that the condition of invariance in the sense of $\left(2^{\circ}\right)$ under the transformation group (B) defined $R_{N}(+)$ and $R^{N}(-)$ is equivalent to the condition that the following relation holds true for all $x$ in $R^{N}(+)$ and $R^{N}(-)$ except for a set of measure zero.

$$
\varphi\left(x_{1}, \ldots, x_{N}\right)=\varphi\left(\frac{x_{1}-x_{N}}{\left|x_{N-1}-x_{N}\right|}, \ldots, \frac{x_{N-2}-x_{N}}{\left|x_{N-1}-x_{N}\right|}, \frac{x_{N-1}-x_{N}}{\left|x_{N-1}-x_{N}\right|}, 0\right) .
$$


The proof of this relation is exactly the same as to that of Theorem 2.1. Then by making use of the transformation

$$
\begin{aligned}
y_{1}= & \left(x_{1}-x_{N}\right) / x_{N-1}-x_{N} \\
& \ldots \\
y_{N-2}= & \left(x_{N-2}-x_{N}\right) / x_{N-1}-x_{N} \\
x= & x_{N-1}-x_{N} \\
z= & x_{N} .
\end{aligned}
$$

Our integral (2.4) will be written in the following sum of two integrals, where the first term of the integrand represents that of the integral on $R^{N}(+)$ and the second on $R^{N}(-)$.

$$
\begin{aligned}
& \int_{y_{1}=-\infty}^{\infty} \int_{y_{2}=-\infty}^{\infty} \ldots \int_{y_{N-2}=-\infty}^{\infty} \int_{x=0}^{\infty} \int_{z=-\infty}^{\infty} x^{N-2} \\
& \left\{\sum _ { i = 1 } ^ { N } a _ { i } f _ { i } \left(y_{1} x+z, y_{2} x+z, \ldots,\right.\right. \\
& \left.y_{N \rightarrow 2} x+z, y_{N-1}+z, z\right) d_{i}\left(y_{1}, y_{2}, \ldots, y_{N \rightarrow-1},+1,0\right) \\
& +\sum_{i=1}^{N} a_{i} f_{i}\left(-y_{1} x+z,-y_{2} x+z, \ldots,\right. \\
& \left.\left.-y_{N-2} x+z,-y_{N-1}+z, z\right) d_{i}\left(y_{1}, y_{2}, \ldots, y_{N-2},-1,0\right)\right\} \\
& d y_{1} d y_{2} \ldots d y_{N \rightarrow 2} d x d z \\
& =\int_{R^{N-2}} \sum_{i=1}^{N} a_{i}\left\{g_{i}\left(y_{1}, \ldots, y_{N-2}\right) d_{i}\left(y_{1}, \ldots, y_{N-2},+1,0\right)\right. \\
& \left.+g_{i}\left(-y_{1}, \ldots,-y_{N-2}\right) d_{i}\left(y_{1}, y_{2}, \ldots, y_{N-2},-1,0\right)\right\} \\
& d y_{1} d y_{2} \ldots d y_{N-2} \text {, }
\end{aligned}
$$

where $g_{i}\left(y_{1}, \ldots, y_{N-2}\right)$ is defined by $(2.6)$.

This integral is maximized if

(2.14) $d_{i}\left(y_{1}, y_{2}, \ldots, y_{N-2},+1,0\right)=1$, then

$$
\begin{aligned}
& a_{i} g_{i}\left(y_{1}, y_{2}, \ldots, y_{N-2}\right) \\
& \quad=\operatorname{Max}_{j}\left\{a_{j} g_{j}\left(y_{1}, \ldots, y_{N-2}\right), \quad a_{j} g_{j}\left(-y_{1}, \ldots,-y_{N-1}\right)\right\}
\end{aligned}
$$

and $\quad d_{i}\left(y_{1}, y_{2}, \ldots, y_{N-2},-1,0\right)=1$, then

$$
\begin{aligned}
& a_{i} g_{i}\left(-y_{1},-y_{2}, \ldots,-y_{N-2}\right) \\
& \quad=\operatorname{Max}_{j}\left\{a_{j} g_{j}\left(y_{1}, \ldots, y_{N-2}\right), \quad a_{j} g_{j}\left(-y_{1}, \ldots,-y_{N-2}\right)\right\}
\end{aligned}
$$

for all $y$ except for a set of measure zero.

By converting this relation into the one in terms of $\underset{\sim}{x}$, we obtain the relation $(2.10)$.

§3. The slippage tests for normal population. At first we need the following

Lemma 3. 1. Let $\left(x_{1}, x_{2}, \ldots, x_{N}\right)$ be independently distributed in normal distribution with the mean values $m_{1}, m_{2,}, \ldots, m_{N}$ and the variances $\sigma_{i}^{2}$ 
such that $\sigma_{i}^{m}=\ldots=\sigma_{i-1}^{m}=\sigma_{i}^{2} / \delta \cdots \sigma_{i+1}^{m}:=\ldots=\sigma_{N}^{2}$ where $\delta \geq 1$ respectively. Let $f_{i}(\underset{\sim}{\boldsymbol{x}})$ be the probability density function of $\underset{\sim}{\boldsymbol{x}}=\left(x_{1}, \ldots, x_{x}\right)$, and $\boldsymbol{g}_{i}(\underset{\sim}{\boldsymbol{y}})$ be the function defined by the relation (2.10). Then we have

$$
\begin{aligned}
& g_{i}\left(\frac{x_{1}-x_{N}}{\left|x_{N-1}-x_{N}\right|}, \ldots, \frac{x_{N-1}-x_{N}}{x_{N-1}-x_{N} \mid}\right) \\
= & \left.C\left|x_{v-1}-x_{N}\right|^{N}\right|_{0} ^{\infty} t^{\nu} \exp [-1 \\
& t^{2}\left\{(1+(\delta-1)(1-1 / N))\left(N \sum_{\nu=1}^{N} x_{\nu}^{2}-\left(\sum_{\nu=1}^{N} x_{\nu}\right)^{2}-N(\delta-1)\left(x_{i}-\bar{x}\right)^{2}\right)\right\} \\
- & 2 t\left\{( 1 + ( \delta - 1 ) ( 1 - 1 / N ) ) \left(N \sum_{\nu=1}^{N} m_{\nu} x_{\nu}-\left(\sum_{\nu=1}^{N} m_{\nu}\right)\left(\sum_{\nu=1}^{N} x_{\nu}\right)\right.\right. \\
+ & \quad\left\{( 1 + ( \delta - 1 ) ( 1 - 1 / N ) ) \left(N \sum_{\nu=1}^{N} m_{\nu}^{2}-\left(\sum_{\nu=1}^{N} m_{\nu}\right)\left(\sum_{\nu=1}^{N} x_{\nu}\right)\right.\right. \\
& \left.\left.\left.-N(\delta-1)\left(m_{i}-\bar{m}\right)^{2}\right)\right\}\right] d t,
\end{aligned}
$$

where $C$ and $R$ are some constants common for all $i$ and $\bar{x}=\sum_{\nu=1}^{N} x_{\nu} / N$ and $\bar{m}=\sum_{\nu=1}^{N} m_{\nu} / N$.

Proof. The proof is immediate and is omitted.

One remark should be made here. The optimum slippage tests for the mean of normal population were treated by E. PAULSON [5] for the case when the sample sizes from each population are the same and are more than one, and also by A. KuDô [3] whose result includes the case when the same are equal to one, and the statistic in this case is nothing but the Pearson-Chandrasekar's statistic for testing the outlying observation. Though the result of the former can be proved in the exactly same way as to that of the latter, the arguments in these two papers can be much simplified by making use of this Lemma above stated.

In this paper, we shall discuss the problem in a somewhat more general formulation. Let $x_{i}$ be independently distributed normally with the mean values $m_{i}$ and variances $\sigma_{i}{ }^{2}(i=1,2, \cdots, N)$. We have $N+1$ hypothesis, namely

$$
\begin{aligned}
H_{0} & =H\left(m_{1}=m_{2}=\ldots=m_{N}, \sigma_{1}{ }^{2}=\sigma_{2}^{2}=\ldots=\sigma_{N}^{2}\right) \\
H_{i} & =H_{i}(\Delta, \delta)=H\left(m_{1}=\ldots=m_{i-1}=m_{i}-\Delta=m_{i+1}=\ldots\right. \\
& \left.=m_{N}, \sigma_{1}{ }^{2}=\ldots=\sigma_{i-1}^{2}=\sigma_{i}{ }^{2} / \delta=\sigma_{i+1}^{2}=\ldots=\sigma_{N}\right) \quad(i=1,2, \ldots, N),
\end{aligned}
$$

where none of these pararmeters are known to us.

Let $D_{i}(i=0,1, \ldots, N)$ be the decision of accepting $H_{i}(i=0,1, \ldots, N)$ and $P_{r}\left(D_{i} / H_{i 1}\right)$ and $P_{r}\left(D_{i} / H_{j}(\Delta, \delta)\right)$ be the probability of taking the decision $D_{i}$. when $H_{0}$ and $H_{j}(\Delta, \delta)$ is correct respectively. Our problem here is to 
find out the most suitable decision procedure as to these $N+1$ decisions. An answer is given in

Theorem 3.1. Among all the decision procedures invariant under the transformation group $(B)$ in the sense of $\left(2^{\circ}\right)$, such that

$$
P_{r}\left(D_{0} / H_{11}\right)=1-\alpha,
$$

where $\alpha$ is a pre-assigned probability and

$$
q(\Delta, \delta)=P_{r}\left(D_{i} / H_{i}(\Delta, \delta)\right) \quad(i=1, \ldots, N)
$$

there exists a decision procedure which maximizes the function and it is given by the following.

In cases $\left(1^{\circ}\right) \Delta>0, \delta=1,\left(2^{\circ}\right) \Delta>0, \delta>1,\left(3^{\circ}\right) \Delta>0, \delta \geq 1$ it is given by the following:

$$
\text { take } D_{0} \text { if } \operatorname{Max}_{j}\left(x_{j}-x\right) / s \leq \lambda_{a}^{(1)}(N)
$$$$
\text { take } D_{i} \text { if } \operatorname{Max}_{j}\left(x_{j}-\bar{x}\right) / s>\lambda_{a}^{(1)}(N) \text { and } \operatorname{Max}_{j} x_{j}=x_{i}
$$

except for a set of measure zero.

In cases $\left(4^{\circ}\right) \Delta<0, \delta=1,\left(5^{\circ}\right) \Delta<0, \delta>1,\left(6^{\circ}\right) \Delta<0, \delta \geq 1$

it is given by the following:

$$
\text { take } D_{0} \text { if } \operatorname{Min}_{j}\left(x_{j}-\bar{x}\right) / s \geq \lambda_{a}^{(2)}(N)
$$

$$
\text { take } D_{i} \text { if } \operatorname{Min}_{j}\left(x_{j}-\bar{x}\right) / s<\lambda_{\alpha}^{(2)}(N) \text { and } \operatorname{Min}_{j} x_{j}=x_{i}
$$

except for a set of measure zero.

In cases $\left(7^{\circ}\right) \Delta=0, \delta>1,\left(8^{\circ}\right) \Delta=0, \delta \geq 1$, it is given by the following:

$$
\begin{array}{llll}
\text { take } & D_{0} & \text { if } & \operatorname{Max}\left|x_{j}-\bar{x}\right| s \leq \lambda_{\alpha}^{(3)}(N) \\
\text { take } & D_{0} \text { if } \operatorname{Max}\left|x_{j}-\bar{x}\right| / s>\lambda_{a}^{(3)}(N) \text { and } \operatorname{Max}_{j}\left|x_{j}-x\right|=\left|x_{i}-\bar{x}\right|
\end{array}
$$
except for a set of measure zero.

Further if we assume a restriction

$$
q(\Delta, \delta)=q(-\Delta, \delta)
$$

the same decision procedure in cases $\left(\mathcal{G}^{\circ}\right) \Delta \neq 0, \delta=1,\left(10^{\circ}\right) \Delta \neq 0, \delta>1$, $\left(11^{\circ}\right) \Delta \neq 0, \delta \geq 1$ is given by the procedure in (3.7).

Here $\bar{x}=\sum_{i=1}^{N} x_{i} / N, s^{2}=\sum_{i=1}^{N}\left(x_{i}-\bar{x}\right)^{2} / N$, and $\lambda_{\alpha}^{(1)}(N), \lambda_{\alpha}^{(2)}(N)$ and $\lambda_{\alpha}^{(3)}(N)$ are some constants depending on $N$ and $\alpha$ only, and when there are more than one observation which take the maximum or the minimum value, we can take any one of the allowable decisions in each case.

Proof. We shall briefly state the proof as it is exactly the same as those in E. PAulson [5] and A. KuDô [3]. 
Let $d_{i}(\underset{\sim}{x})(i=0,1, \ldots, N)$ denote the probability of taking decisions $D_{i}$ $(i=0,1, \ldots, N)$ respectively after observing $x=\left(x_{1}, x_{2}, \ldots, x_{v}\right)$, namely the decision function as to these $N+1$ diecisions, and let $f_{0}(\underset{\sim}{x})$ and $f_{i}(\underset{\sim}{x} / \Delta, \delta)$ be the frequency functions of $x$ when $H_{0}$ and $H_{i}(\Delta, \delta)$ are correct respectively. Then we can easily see that for any $\Delta$ and $\delta$, we can always find a positive constant $A$ such that the decision function expressing the decision procedure given in this theorem maximizes the integral

$$
\int A d_{0}(\underset{\sim}{x}) f_{0}(\underset{\sim}{x})+\sum_{i=1}^{N} d_{i}(\underset{\sim}{x}) f_{i}(/ \underset{\sim}{x} \Delta, \delta) d x
$$

among all the decision functions which express the decision procedures satiafying the assumptions of this theorem in any one of the cases. And this fact will lead as to the conclusion.

It may be noticed here that this theorem contains the result of the author in the previous paper [3] as its special case $\delta=1$, and also that we are making use of Pearson-Chandrasekar's statistic in the procedures given in (3.5) and (3.6).

§4. The slippage tests for the exponential and $\chi^{2}$ distributions. In this section we shall be concerned with the gamma distribution, with the location parameter equal to zero, namely the frequency function is given by

$$
f(x / \lambda, a)= \begin{cases}0 & x \leq 0 \\ a^{\lambda} x^{\lambda-1} e^{-a x} / \Gamma(\lambda) & x>0 .\end{cases}
$$

At first we have the following

Lemma 4.1. If we define

$$
f\left(x_{1}, \ldots, x_{N}\right)=\prod_{i=1}^{N} a_{i}^{{ }^{\lambda i}} x_{i}^{\lambda_{i-1}} e^{-a_{i} x_{i}} / \Gamma\left(\lambda_{i}\right)
$$

then the function defined by (2.9) is given by

and

$$
\begin{aligned}
& g\left(y_{1}, \ldots, y_{N-1}\right) \\
& \quad=\Gamma\left(\sum_{i=1}^{N} \lambda_{i}\right)\left(\prod_{i=1}^{N} a_{i}^{\lambda_{i}}\right)\left|a_{1} y_{1}+\ldots+a_{N-1} y_{N-1}+a_{v}\right|^{\lambda_{N}} / \prod_{i=1}^{N} \Gamma\left(\lambda_{i}\right)
\end{aligned}
$$

$$
\begin{aligned}
& \boldsymbol{g}\left(\frac{x_{1}}{\left|x_{N}\right|}, \ldots, \frac{x_{N-1}}{\left|x_{N}\right|}\right) \\
& \quad=\Gamma\left(\sum_{i=1}^{N} \lambda_{i}\right)\left(\prod_{i=1}^{N} a_{i}^{\lambda_{i}}\right)\left|x_{N}\right|^{\lambda_{N}}\left|a_{1} x_{1}+\ldots+a_{N} x_{N}\right|^{\lambda_{N}} / \prod_{i=1}^{N} \Gamma\left(\lambda_{i}\right) .
\end{aligned}
$$

Proof. The proof is quite simple and is omitted.

It may be mentioned here that in case $a=1 / 2$ and $\lambda=n / 2,(4.1)$ is the frequency function of the $\chi^{2}$-distribution with $n$ degree of freedom, and 
further this will enable us to give a shorter proof of the result obtianed by TRAUX [7], which states the optimum property of the statistic introduced by Cochron concerning the slippage test for the variances of $k$ normal populations [1].

In case $a=1 / m, \lambda=1$, we have the frequency function of the exponential distribution, i.e. $\boldsymbol{F}(\boldsymbol{x})=1-e^{-x^{\prime} m}$ and in this case also there arises a problem of slippage test.

Let $\left\{x_{i j}\right\}\left(i=1,2, \ldots, i ; j=1,2, \ldots, n_{i}\right)$ be independently distributed in exponential distribution, where for each $i, n_{i}$ random variables $\left(x_{i 1}, \ldots, x_{i n_{i}}\right)$ are of the same parameter $m_{i}$. Here we have the same sort of hypothesis

$$
\begin{aligned}
& H_{11}=H\left(m_{1}=m_{2}=\ldots=m_{k}\right) \\
& H_{i}=H_{i}(\delta)=H\left(m_{1}=\ldots,=m_{i-1}=m_{i} / \delta=m_{i+1}=\ldots=m_{k}\right) \\
& \quad(i=1,2, \ldots, k),
\end{aligned}
$$

where these parameters are unknown to us and we have,

Theorem 4.1. In the same notations as above, we have that among all the decision procedures invariant under the transformation group $(D)$ in the sense of $\left(2^{\circ}\right)$, such that

$$
P_{r}\left(D_{01} / H_{0}\right)=1-\alpha,
$$

where $\alpha$ is a pre-assigned probability, and

$$
q(\delta)=P_{r}\left(D_{i} / H_{i}(\delta)\right) \quad(i=1,2, \ldots, k)
$$

there exists a decision procedure which maximizes the function and it is given by the following

In case $\left(1^{\circ}\right) \delta>1$

take $D_{n}$ if $\operatorname{Max}_{i} \sum_{j=1}^{n_{i}} x_{i j} / \sum_{i=1}^{k} \sum_{j=1}^{n_{i}} x_{i j}<\mu_{\alpha}^{(1)}\left(n_{1}, \ldots, n_{k}\right)$

take $D_{1}$ if $\operatorname{Max}_{i} \sum_{j=1}^{n_{i}} \boldsymbol{x}_{i j} / \sum_{i=1}^{k} \sum_{j=1}^{n_{i}} x_{i j}>\mu_{\alpha}^{(1)}\left(\boldsymbol{n}_{1}, \ldots, n_{k}\right)$,

$$
\operatorname{Max}_{i} \sum_{j=1}^{n_{i}} x_{i j}=\sum_{j=1}^{n_{l}} x_{l j},
$$

and in case $\left(2^{\circ}\right) \delta<1$

take $D_{0}$ if $\operatorname{Min}_{i} \sum_{j=1}^{n_{i}} x_{i j} / \sum_{i=1}^{k} \sum_{j=1}^{n_{i}} x_{i j}>\mu_{\alpha}^{(2)}\left(n_{1}, \ldots, n_{k}\right)$

take $D_{l}$ if $\operatorname{Min}_{j} \sum_{i=1}^{n_{i}} x_{i, j} / \sum_{i=1}^{k} \sum_{j=1}^{n_{i}} x_{i j}<\mu_{\alpha}^{(2)}\left(n_{1}, \ldots, n_{k}\right)$,

$$
\operatorname{Min}_{i} \sum_{j=1}^{n_{i}} x_{i j}=\sum_{j=1}^{n_{l}} x_{l j},
$$

except for a set of probability zero, where $\mu_{\alpha}^{(1)}\left(n_{1}, \ldots, n_{k}\right)$ and $\mu_{\alpha}^{(2)}\left(n_{1}, \ldots, n_{k}\right)$ are the constants depending on $\alpha$ and $n_{i}$ 's, and when there are more than 
one observations which take the maximum or the minimum value, we can take any one of the allowable decisions in each case.

Proof. The proof is the exactly same as to that of Theorem 3.1.

Concerning the calculation of $\mu_{\alpha}^{(1)}\left(\boldsymbol{n}_{1}, \ldots, \boldsymbol{n}_{k}\right)$ and $\mu_{\alpha}^{(2)}\left(\boldsymbol{n}_{1}, \ldots, \boldsymbol{n}_{k}\right)$ the usual method would be the approximation formulae by making use of the BONFERnI's theorem ( $c f$. p. 75 of FELLER [2]). Here we shall state only the special case when $n_{1}=n_{2}=\ldots=n_{k}=n$.

Theorem 4.2. Let $\mu_{\alpha}^{(1)}(n, k)$ and $\mu_{\alpha}^{(2)}(n, k)$ denote $\mu_{a}^{(1)}\left(n_{1}, n_{2}, \ldots, n_{k}\right)$ and $\mu_{\alpha}^{(2)}\left(n_{1}, n_{2}, \ldots, n_{k}\right)$ when $n_{1}=n_{2}=\ldots=n_{k}=n$, then we have approximately,

$$
\frac{p}{k} \cong 1-I_{\mu_{\alpha}(1)_{(n, k)}}(n, n(k-1))
$$

and

$$
\frac{p}{k} \cong I_{\mu_{\alpha}(2)(n, k)}(\boldsymbol{n}, \boldsymbol{n}(k-1)),
$$

where $I_{u}(p, q)$ is the incomplete Beta-function

$$
I_{u}(p, q)=\frac{1}{B(p, q)} \int_{0}^{u} x^{p-1}(1-x)^{q-1} d x
$$

Proof. As each of the $\sum_{j=1}^{n} x_{i j}$ is distributed in the gamma distribution with the density function

$$
\left(\frac{1}{m_{i}}\right)^{n} e^{-\frac{x}{m_{i}}} x^{n-1} / \Gamma(n)
$$

we have, after simple calculations, under the null hypothesis

$$
P_{r}\left(\frac{\sum_{i=1}^{n} x_{i j}}{\sum_{i=1}^{k} \sum_{j=1}^{n} x_{i j}}<\lambda\right)=I_{\lambda}(n, n(k-1))
$$

for all $i$. By applying the BONFERn's theorem, we immediately get (4.11) and (4.12).

\section{KyUSYU UNIVERSITY}

\section{References}

[1] Cochran, W. G.; The distribution of the largest of a set of estimated variances as a fraction of their total. Ann. Eug., 11 (1941), 47-52.

[2] Fellek, W.; An introduction to probability theory and its applications. Vol. 1. John wiley \& Sons, Inc., New York, 1950. 
[3] Kudô, A.; On the testing of outlying observations. Submitted to Sankhya.

[4] Lemanas, E. L.; Some principles of the theory of testing hypothesis. Ann. Math. Stat., 21 (1950), 1-26.

[5] Paurson, E.; An optimum solution to the $k$ sample slippage problem for the normal distribution. Ann. Math. Stat., 23 (1952), 610-616.

[6] Pearson, E. S. and Chandra Sekar, C.; The efficiency of statistical tools and a criterion for the rejection of outlying observations. Biometrika, 28 (1936), 308-320.

[7] Truax, D. R.; An optimum slippage test for the variances of $k$ normal disiributions. Ann. Math. Stat., 24 (1953), 47-52.

[8] Wald, H.; Statistical decision functions. John Wiley \& Sons, Inc., New York, 1950. 